\title{
LA REALIDAD AUMENTADA COMO HERRAMIENTA DE ENRIQUECIMIENTO DEL PROCESO DE APRENDIZAJE
}

\author{
AUGMENTED REALITY AS A TOOL FOR ENRICHING \\ THE LEARNING PROCESS
}

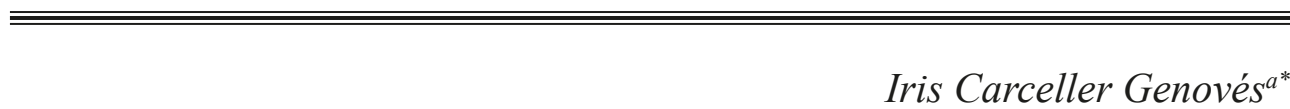

Fechas de recepción y aceptación: 18 de julio de 2019, 6 de diciembre de 2019

Resumen: La sociedad evoluciona y, por ello, el proceso de enseñanza-aprendizaje debe estar preparado para esa evolución y ofrecer al alumnado las herramientas suficientes para enfrentarse a ella. Por ello, desde este artículo se pretende dar una visión general a los beneficios que puede aportar la realidad aumentada al proceso de aprendizaje, es decir, se trata de demostrar que la realidad aumentada es una ventana abierta al mundo, que nos ofrece un sinfín de posibilidades para acercar a las escuelas la realidad que nos rodea. A lo largo de este trabajo se pretende demostrar que la realidad aumentada, junto con diferentes metodologías emergentes, es una herramienta necesaria en las escuelas para poder enriquecer el proceso de aprendizaje y llevar a cabo un proceso más real y vivencial para el alumnado. La inclusión de la tecnología de la información y la comunicación en las aulas es de vital importancia, ya que nos facilita la presentación de los contenidos y los materiales de diferentes formas para poder llegar a todos y cada uno de nuestros alumnos y convertir la enseñanza en un proceso de aprendizaje para todos, es decir, en un proceso que ofrezca a cada alumno lo que necesita. Estas ofrecen la oportunidad de romper la brecha digital existente entre los diferentes grupos sociales que se encuentran en la escuela. La realidad aumentada ayuda a acercar al alumno la realidad que le rodea de una manera más concreta y vivencial para que así pueda entender el mundo en el que vive.

${ }^{a}$ Ayuntamiento de Valencia. Colegio Municipal Profesor Santiago de Grisolia.

* Correspondencia: Colegio Municipal Profesor Santiago de Grisolia. Calle Ramón de Campoamor, 89. 46022 Valencia. España.

E-mail: icarceller@valencia.es 
Palabras clave: realidad aumentada, tecnología de la información y la comunicación, brecha digital, educación inclusiva.

Abstract: Society evolves, and so the teaching-learning process must be prepared for this evolution and provide students with sufficient tools to confront it. Therefore, the aim of this article is to give an overview of the benefits that augmented reality can bring to the learning process, that is, to show that augmented reality is a window into the world, which offers us endless possibilities to bring the reality that surrounds us to schools. Throughout this work we intend to demonstrate that augmented reality, together with different emergent methodologies, is a necessary tool in schools to enrich the learning process and to carry out a more real and experiential process for students. The inclusion of information and communication technology in classrooms is of vital importance, because it makes it easier for us to present the contents and materials in different ways and so we can reach each and every one of our students and turn teaching into a learning process for all, i. e., into a process that offers each student what he needs. These technologies provide an opportunity to bridge the digital gap between the different social groups in school. Augmented reality helps to bring the student closer to the reality that surrounds him in a more concrete and experiential way so that he can understand the world in which he lives.

Keywords: augmented reality, information and communication technology, digital gap, inclusive education.

\section{INTRODUCCIÓN}

Para profundizar en la investigación se han definido tres conceptos de vital importancia en el proyecto: realidad mixta, realidad virtual y realidad aumentada.

La realidad mixta consiste en combinar mundos virtuales con el mundo real (físico) a tiempo real. Esta combinación permite crear nuevos espacios en los que interactúan tanto objetos y/o personas reales como virtuales.

Milgram y Kishino (1994) definieron la realidad mixta como cualquier espacio entre los extremos del continuo de la virtualidad. Este continuo de la virtualidad se extiende desde el mundo completamente real hasta el entorno completamente virtual, encontrándose entre medio de estos la realidad aumentada y la realidad virtual.

Por ello, es de vital importancia que se distinga entre realidad virtual y la realidad aumentada, ya que gracias a estas dos realidades creamos la llamada realidad mixta. 
Así, se entiende la realidad virtual (RV) como el nombre dado a un conjunto de técnicas y tecnologías basadas en ordenador, que aproxima la visualización de conceptos, objetos y acciones en tres dimensiones (3D) de una forma interactiva, asemejándose a la realidad.

La realidad virtual (RV) describe un entorno que es simulado por ordenador. Muchos entornos de realidad virtual son principalmente experiencias visuales mostradas en una pantalla o a través de unas gafas espectroscópicas, que pueden incluir información sensorial adicional, como sonido.

El término fue acuñado por Jaron Lanier (1989). Lanier, uno de los pioneros en este campo, fundó la empresa VPL Research (Virtual Programming Languages), con la que creó algunos de los primeros sistemas de RV en la década de 1980.

Por último, y como eje fundamental de la investigación, se entiende la realidad aumentada (RA) como la combinación de lo real y lo virtual en tiempo real y la ubicación en el mismo sistema de coordenadas 3D que la realidad; es decir, hace referencia a la visualización directa o indirecta de elementos del mundo real combinados o aumentados con elementos virtuales generados por un ordenador, cuya fusión da lugar a la realidad mixta (Cobo y Moravec, 2011).

La RA es un sistema tecnológico que está en desarrollo. Se trata de una tecnología emergente. En términos coloquiales, entendemos la RA como un sistema que potencia las capacidades de nuestros sentidos, mediante la unión de elementos reales y elementos virtuales.

Según Azuma (1994) la RA es un entorno que incluye elementos de realidad virtual y aspectos del mundo real. También es definida por Cabero (2013), Gómez (2013), Cabero y Barroso, (2015, 2016a, 2016b), Cabero y García (2016) y Cabero, Leiva, Moreno, Barroso y López (2016) como aquel entorno en el que tiene lugar la integración de lo virtual y lo real, es decir, la combinación de información digital e información física en tiempo real a través de distintos dispositivos tecnológicos; o lo que es lo mismo, la RA consiste en utilizar un conjunto de dispositivos tecnológicos que añaden información virtual a la información física, para crear de esta forma una nueva realidad, en la cual, no obstante, tanto la información real como la virtual desempeñan un papel significativo para la construcción de un nuevo entorno comunicativo mixto amplificado y enriquecido. Otros autores elaboran conceptos más 
complejos abarcando más elementos implicados en este proceso. Como nos indican De Pedro (2011) y Prendes (2015), debemos tener en cuenta que la RA no reemplaza el mundo real por uno virtual, como ocurre si empleamos tecnología basada en la realidad virtual, sino al contrario, la realidad aumentada nos permite mantener el mundo real que ve el usuario complementándolo con información virtual que se superpone sobre la real. De ese modo, el usuario recibe estímulos del contexto real y del contexto virtual, amplificándose las posibilidades de aprendizaje.

Por último, diversos autores (Basogain, Olabe, Espinosa, Rouèche y Olabe, 2007; Kato, 2010) indican que la RA se puede definir como objetos virtuales o anotaciones que pueden ser superpuestos en el mundo real como si realmente existieran integrados en el entorno físico, es decir, esta tecnología mantiene el mundo real complementándolo con información virtual.

Existen varios autores que especifican diferentes niveles de RA (Estebanell, Ferrés, Cornellà y Codina, 2012; Lens-Fitzgerald, 2009; Reinoso, 2012; Rice, 2009). Se pueden entender los niveles como una forma de medir la complejidad de las tecnologías involucradas en el desarrollo de sistemas de RA. En principio, a más nivel, mayores son las posibilidades de las aplicaciones. Estos niveles son:

- Nivel 0. Hiperenlazando el mundo físico (physical world hyper linking). Basado en códigos de barra (enlaces 1D, Universal Product Code), códigos 2D (por ejemplo, los códigos $\mathrm{QR}$ ) o reconocimiento de imágenes aleatorias. Lo característico de este nivel 0 es que los códigos son hiperenlaces a otros contenidos, no existe registro en $3 \mathrm{D}$ ni seguimiento de los marcadores (básicamente funcionan como un hiperenlace HTML pero sin necesidad de teclear).

- Nivel 1. Realidad aumentada basada en marcadores (marker based AR). Normalmente supone el reconocimiento de patrones 2D; el reconocimiento 3D de objetos (por ejemplo, una silla) sería la forma más avanzada de nivel 1 de RA. Según Estebanell et al. (2012), "los marcadores son unas imágenes en blanco y negro, generalmente cuadradas, con dibujos sencillos y asimétricos".

- Nivel 2. RA sin marcadores (markerless AR). Mediante el uso del GPS y la brújula de los dispositivos electrónicos, conseguimos localizar la 
situación y la orientación y superponer POI (puntos de interés) en las imágenes del mundo real. Lens-Fitzgerald (2009) lo define como AR basada en GPS-brújula. También puede incluir el uso de acelerómetros para calcular la inclinación.

- Nivel 3. Visión aumentada. Citando a Rice (2009): "Debemos despegarnos del monitor o el display para pasar a ligeros, transparentes displays para llevar encima (de una escala como las gafas). Una vez la RA se convierte en VA (visión aumentada), es inmersiva. La experiencia global inmediatamente se convierte en algo más relevante, contextual y personal. Esto es radical y cambia todo”. Este nivel no está todavía disponible.

Por lo tanto, la RA se aplica a un tipo de realidad mixta formada por la integración coherente de la realidad física y un tiempo real de una capa de información digital que puede ser diversa (texto, símbolo, audio, vídeo y/u objetos tridimensionales) y con la que es posible la interacción, con el resultado de enriquecer o alterar la información de la realidad física en la que se integra.

La RA tiene como propiedades principales:

- Realidad mixta.

- Integración coherente en tiempo real: mismas coordenadas espaciotemporales para todos los elementos.

- Diversidad de la capa de información digital.

- Posibilidad de interacción.

- Enriquecimiento o alteración de la información.

\section{Desarrollo}

El proyecto que se presenta está dirigido al alumnado de Educación Primaria. Se propone un proyecto transversal llamado "Vivimos en la Ciudad", para dar a conocer los distintos tipos de ciudades que existen, así como sus sociedades y culturas, entre otros factores. 
Los objetivos de la propuesta se encuentran en el Real Decreto 126/2014, el cual establece el currículo de Educación Primaria, en su artículo 7 y en la LOMCE en su artículo 17.

Se exponen los objetivos generales para la etapa educativa de la Educación Primaria:

a) Conocer y apreciar los valores y las normas de convivencia, aprender a obrar de acuerdo con ellas, prepararse para el ejercicio activo de la ciudadanía y respetar los derechos humanos, así como el pluralismo propio de una sociedad democrática.

b) Desarrollar hábitos de trabajo individual y de equipo, de esfuerzo y de responsabilidad en el estudio, así como actitudes de confianza en uno mismo, sentido crítico, iniciativa personal, curiosidad, interés y creatividad en el aprendizaje, y espíritu emprendedor.

c) Adquirir habilidades para la prevención y para la resolución pacífica de conflictos, que les permitan desenvolverse con autonomía en el ámbito familiar y doméstico, así como en los grupos sociales con los que se relacionan.

d) Conocer, comprender y respetar las diferentes culturas y las diferencias entre las personas, la igualdad de derechos y oportunidades de hombres y mujeres y la no discriminación de personas con discapacidad.

e) Conocer y utilizar de manera apropiada la lengua castellana y, si la hubiere, la lengua cooficial de la comunidad autónoma y desarrollar hábitos de lectura.

f) Adquirir en al menos una lengua extranjera la competencia comunicativa básica que permita expresar y comprender mensajes sencillos y desenvolverse en situaciones cotidianas.

g) Desarrollar las competencias matemáticas básicas e iniciarse en la resolución de problemas que requieran la realización de operaciones elementales de cálculo, conocimientos geométricos y estimaciones, así como ser capaces de aplicarlos a las situaciones de su vida cotidiana.

h) Conocer los aspectos fundamentales de las ciencias de la naturaleza, las ciencias sociales, la geografía, la historia y la cultura. 
i) Iniciarse en la utilización, para el aprendizaje, de las tecnologías de la información y la comunicación, desarrollando un espíritu crítico ante los mensajes que reciben y elaboran.

j) Utilizar diferentes representaciones y expresiones artísticas e iniciarse en la construcción de propuestas visuales y audiovisuales.

k) Valorar la higiene y la salud, aceptar el propio cuerpo y el de los otros, respetar las diferencias y utilizar la educación física y el deporte como medios para favorecer el desarrollo personal y social.

l) Conocer y valorar los animales más próximos al ser humano y adoptar modos de comportamiento que favorezcan su cuidado.

$m$ ) Desarrollar sus capacidades afectivas en todos los ámbitos de la personalidad y en sus relaciones con los demás, así como una actitud contraria a la violencia, a los prejuicios de cualquier tipo y a los estereotipos sexistas.

n) Fomentar la educación vial y actitudes de respeto que incidan en la prevención de los accidentes de tráfico.

Hemos elegido todos los objetivos que los alumnos de la Educación Primaria deben alcanzar al acabar esta etapa porque nuestra propuesta engloba toda la etapa. Se entiende que dicha intervención tiene carácter transversal a lo largo de la Educación Primaria, ya que abarca todas las áreas de conocimiento.

En la Ley Orgánica de mejora educativa 8/2013, de 9 de diciembre, y en el Real Decreto 126/2014, de 28 de febrero, el cual establece el currículo de Educación Primara, se establecen siete competencias clave.

También se han tenido en cuenta las competencias clave como las destrezas y actitudes que todos los alumnos necesitan para su realización y desarrollo personal, para su inclusión en la sociedad y para su incorporación en el mundo de la ocupación.

Estas siete competencias son:

- Comunicación lingüística.

- Competencia matemática y competencias básicas en ciencia y tecnología.

- Competencia digital. 
- Aprender a aprender.

- Competencias sociales y cívicas.

- Sentido de iniciativa y espíritu emprendedor.

- Conciencia y expresiones culturales.

Los contenidos de nuestra propuesta didáctica los encontramos en el Decreto 108/2014, de 4 de julio, del Consell, por el que establece el currículo y desarrolla la ordenación general de la Educación Primaria en la Comunitat Valenciana.

Todos los contenidos están relacionados con el eje temático de nuestro proyecto "Vivimos en la ciudad", temática que irá desde la máxima cercanía a lo más lejano con respecto al alumno. En primer ciclo los alumnos tendrán contenidos relacionados con "Mi ciudad", en segundo ciclo, se centrarán en "Ciudades de España" y en tercer ciclo, en "Ciudades de Europa".

La metodología de nuestra propuesta se basa en los principios de inclusión educativa:

- Principio de equidad.

- Principio de cooperación.

- Principio de solidaridad.

Los indicadores de inclusión en los que nos centraremos son:

- Los estudiantes se ayudan unos a otros.

- Los miembros del personal de la escuela colaboran entre ellos.

- El personal de la escuela y el alumnado se tratan con respeto.

- Existe relación entre el personal y las familias.

- El personal de la escuela y los miembros del Consejo Escolar (comunidad educativa) trabajan bien juntos.

- Todas las instituciones de la localidad están involucradas en la escuela.

- Todo el mundo se siente acogido.

- Se tienen altas expectativas respecto de todo el alumnado.

- El personal, el alumnado, las familias y los miembros de la comunidad educativa comparten la misma filosofía inclusiva.

- Se valora de igual manera a todos los alumnos y alumnas. 
- El personal de la escuela y los alumnos son tratados como personas y poseedores de un "rol".

- El personal de la escuela intenta eliminar todas las barreras para el aprendizaje y la participación.

- La escuela se esfuerza en disminuir las prácticas discriminatorias.

Por otro lado, la propuesta está basada en un aprendizaje basado por proyectos. Dicha metodología va a ser el eje vertebral de nuestra propuesta, ya que todo el centro colabora en elaborar el proyecto dedicado a "Vivimos en la ciudad". Cada ciclo elaborará sus propios proyectos y actividades, así como la evaluación de dichos trabajos, para poder acercarlos a la realidad que les rodea y aplicarlos en esta. Todo ello se realizará a través de agrupamientos heterogéneos divididos en tres grandes grupos: primero y segundo de primaria trabajarán juntos, así como tercero y cuarto y quinto y sexto, es decir, se intentará formar agrupamientos con alumnado de las diferentes parejas de niveles educativos que se han mencionado.

También cabe decir que se deben realizar actividades en todas las áreas curriculares, pertenecientes a la etapa de la Educación Primaria, ya que se pretende un aprendizaje global.

Siguiendo con la línea de redacción, se debe saber que para llevar a cabo una programación se necesitan recursos tanto materiales, humanos como organizativos, los cuales deben estar de acuerdo con las actividades que realizar, así como con las posibilidades del centro. Estas actividades deben ser atractivas, ayudándose de los recursos necesarios para alcanzar el objetivo fundamental: "que los alumnos disfruten aprendiendo".

Por otro lado, los recursos materiales los hemos dividido en tres grandes grupos: recursos de realidad aumentada, recursos TIC y recursos tradicionales.

Contamos, en primer lugar, con los recursos de realidad aumentada:

1. Códigos QR.

2. HP Reveal/Wallame.

3. DibuPrint.

4. Impresora 3D.

5. Thingervers. 
6. Thinkcards.

7. Programa para la impresora 3D.

8. Geo Aumentaty.

9. ARCrowd.

10. Creator.

11. Sketchup Make.

En segundo lugar, los recursos TIC son:

1. Tabletas.

2. Ordenadores.

3. Impresora.

4. Moodle.

5. Fast Scanner.

6. Lector códigos QR.

7. Páginas web.

Por último, los recursos organizativos hacen referencia al tiempo y al uso del espacio del cual disponemos. Por lo que se refiere al tiempo, contamos con todo el segundo y el tercer trimestre de cada área, ya que se pretende que en el primer trimestre los alumnos se familiaricen con la realidad aumentada y en el segundo, que estos lleven a cabo todo el proyecto.

Otra parte de los recursos organizativos es el espacio del que disfrutamos para las sesiones. Se necesitan diferentes espacios para su realización. Entre ellos se cuenta con:

1. Aulas.

2. Patio del centro.

3. Ciudad (ayuntamiento, biblioteca, casa de la cultura...).

4. Aula de música y plástica.

5. Laboratorio de lenguas.

La evaluación se define como la emisión de un juicio acerca de la idoneidad del proceso de enseñanza-aprendizaje, así como otros aspectos más 
generales de la institución escolar, por lo que evaluar es inseparable del acto de enseñar (Camilloni, 1998).

La evaluación se configura como una actividad continua e integrada en el proceso que se lleva a cabo en la escuela, que pretende adecuar la enseñanza al proceso real de aprendizaje del alumnado y no clasificarlos en función de los resultados obtenidos (artículo 20, LOMCE).

Este proceso debe ser individualizado, ya que cada alumno tiene sus propias necesidades y es de vital importancia tenerlas en cuenta dentro del proceso de enseñanza-aprendizaje

Por otro lado, hemos de tener presente que la evaluación debe caracterizarse por ser continua, procesual y formativa. Por ello, nosotros realizaremos una evaluación inicial, una evaluación continua y procesual y una evaluación formativa.

En nuestro proceso de evaluación los alumnos también formarán parte de él, ya que pondremos en marcha la coevaluación. Este sistema de evaluación consiste en que los alumnos evaluarán los trabajos de sus compañeros mediante rúbricas.

Los alumnos aprenderán a evaluar a sus compañeros de forma objetiva, ya que formará parte de la actividad en sí misma y será un paso más para que los estudiantes sean conscientes del proceso de enseñanza-aprendizaje.

Por otro lado, los criterios e indicadores de aprendizaje irán reflejados en cada unidad didáctica dependiendo del ciclo y el área. Estos criterios y estándares están basados en el Decreto 108/2014, de 4 de julio, del Consell, por el que se establece el currículum y despliega la ordenación general de la Educación Primaria en la Comunitat Valenciana.

\section{MÉTOdO}

Para realizar esta investigación se han llevado a cabo diferentes investigaciones en distintas bases de datos, como EBSCO, Google Académico y Web of Science (WOS). Se han utilizado las palabras clave tales como realidad aumentada, tecnología de la información y comunicación y educación primaria. Para ello, se ha utilizado el operador booleano and para encontrar documentos que enmarcaban estos conceptos conjuntamente. 
Por otro lado, se ha realizado un análisis legislativo relacionado con las diferentes leyes educativas que rigen la Educación Primaria en aspectos como el currículum, la evaluación, el fomento lector y los elementos transversales.

\section{Conclusiones}

Lo que se pretende con esta propuesta es dar a conocer no solo la sociedad, costumbres o arquitectura de una ciudad de nuestro entorno más cercano, sino ampliar el concepto de ciudad a todo aquello que le es desconocido al alumnado.

Para conseguir todo ello, se necesita de un lenguaje de comunicación diferente al que hasta hora conocen los alumnos, la realidad aumentada. Gracias a ella encontramos diferentes recursos para mostrar, de una forma más cercana y real, los contenidos que pretendemos enseñar a nuestro alumnado.

Por otro lado, hacer partícipes a los alumnos de su propio proceso de aprendizaje es de vital importancia para la realización de este proyecto. Esto se ha conseguido a través de dos vías fundamentales:

- El trabajo en grupo para la generación de contenidos destinados a otros compañeros.

- La utilización de la coevaluación, en la que los alumnos son conscientes de la evaluación que se realiza de los trabajos, así como de los ítems que hay que tener en cuenta para su realización, haciendo que los alumnos centren más los puntos de interés de los proyectos.

Todas las actividades plasmadas en esta propuesta giran en torno a la ciudad, y cada actividad dentro de cada área va de menor a mayor complejidad, pero siempre relacionadas entre sí, ya que los alumnos deben entender el proceso de crecimiento de la ciudad así como su entorno social, natural y cultural.

A lo largo del proyecto, se le da vital importancia a los agrupamientos heterogéneos y a principios como el de participación y colaboración, ya que se pretende que el alumnado esté en todo momento unido entre ellos, al ser una manera de conocer la realidad desde diferentes puntos de vista y conocer 
culturas de diferentes lugares cercanas a ellos, es decir, conocer a las personas que conviven en el mismo centro, así como su forma de interpretación y representación de la información y el conocimiento.

Esta propuesta ofrece la oportunidad de demostrar lo importante que es adaptar los contenidos que pretendemos que los alumnos asuman a las necesidades del centro y a sus características particulares, porque no todos los centros son iguales, ni las familias, ni los alumnos.

\section{BiBLIOGRAFÍA}

Alegría, M. (2015). Aplicaciones de la realidad aumentada en el ámbito de la enseñanza superior. Diseño de un proyecto piloto. Cuadernos de Gestión de Información, 18-35.

Cabero J. y Barroso, J. (2016a). Posibilidades educativas de la realidad aumentada. New Approaches in Educational Research 5(1), 46-52.

CABERo, J. (2013). E-Learning 2.0. III Congreso Internacional sobre Buenas Prácticas con TIC en la Investigación y la Docencia. Universidad de Málaga.

Cabero, J. y Barroso, J. (2016b). Ecosistema de aprendizaje con realidad aumentada. TCE: Tecnología, Ciencia y Educación 5, 141-154.

Cabero, J. y GarcíA, F. (coords.) (2016). Realidad aumentada. Tecnología para la formación. Madrid: Síntesis.

CABERo, J. (1998) Impacto de las nuevas tecnologías de la información y la comunicación en las organizaciones educativas. En Lorenzo, M. et al. (coords.), Enfoques en la organización y dirección de instituciones educativas formales y no formales (pp. 197-206). Granada: Grupo Editorial Universitario.

CABero, J. (2007). Nuevas Tecnologías Aplicadas a la Educación. Madrid: McGraw-Hill.

Cabero, J. (2016). Realidad aumentada: tecnología para la formación. Madrid: Síntesis.

Cabero, J. y Barroso, J. (2015). Realidad Aumentada: posibilidades educativas, en J. Ruiz-Palmero, J. Sánchez-Rodríguez y E. Sánchez-Rivas (eds.), Innovaciones con tecnologías emergentes. Málaga: Universidad de Málaga. 
Cabero, J., Leiva, J. J., Moreno, N. M., Barroso, J. y López, E. (2016). Realidad Aumentada y Educación. Innovación en contextos formativos. Barcelona: Octaedro.

Cabezudo, N. (2011). Inclusión digital: perspectiva y experiencias. Prensas Universitarias de Zaragoza: Zaragoza.

Carvin, Andy (2000). Beyond Access: Understanding the Digital Divide. Conferencia en la Universidad de Nueva York, EE. UU. Recuperado de: http://www.benton.org/Divide/thirdact/speech.html.

Norris, Pippa (2001). Digital Divide, Civic Engagement, Information Poverty and the Internet Worldwide. Cambridge University Press.

Claro, M. (2010). Impacto de las TIC en los aprendizajes de los estudiantes. Estado del arte. CEPAL-Colección Documentos de Proyectos 339 (LC/W.339). Santiago de Chile.

Cobo, C. y Moravec, J. W. (2011). Aprendizaje invisible. Hacia una nueva ecología de la educación. Barcelona: Laboratori de Mitjans Interactius/ Publicacions i Edicions de la Universitat de Barcelona, Col.lecció Transmedia XXI. Recuperado de: http://www.aprendizajeinvisible.com/es/.

DeCreto 108/2014, de 4 de julio, del Consell, por el que establece el currículo y desarrolla la ordenación general de la educación primaria en la Comunitat Valenciana.

Estebanell, M., Ferrés, J., Cornellà, P. y Codina, D. (2012). Realidad aumentada y códigos $Q R$ en educación, en J. Hernández, M. Pennesi, D. Sobrino y A. Vázquez (coords), Tendencias emergentes en educación con TIC. Barcelona: Editorial Espiral.

Lens-Fitzgerald, M. (2009).Augmented Reality Hyper Cycle. Recuperado de: http://www.sprxmobile.com/the-augmented-realityhype-cycle.

Martínez, E., Ascencio, I. y Serrano, A. (2005). Entendiendo y definiendo la brecha digital. Revista RED.

Milgram, P. y Kishino, F. (1994). “A Taxonomy of Mixed Reality Visual Displays". IEICE Transactions on Information and Systems E77-d (12), 13211329.

Mullen, T. (2012) Realidad Aumentada: crea tus propias aplicaciones. Madrid: Anaya. 
Norris, P. (2001). Digital divide. Civil engagement, information poverty and the Internet world wide. Cambridge, Massachusetts: Cambridge University Press.

Reinoso, R. (2012). Posibilidades de la realidad aumentada en educación, en J. Hernández, M. Pennesi, D. Sobrino y A. Vázquez (coords), Tendencias emergentes en educación con TIC (pp. 357-400). Barcelona: Editorial Espiral.

Rice, R. (2009). Augmented vision and the decade of ubiquity. Recuperado de: http://curiousraven.com/future-vision/2009/3/20/augmented-visionand-the-decade-ofubiquity.html.

Rose, D. H. y Meyer, A. (2000). The Future is in the Margins: The Role of Technology and Disability in Educational Reforms. Center for Applied Special Technology. Recuperado de: http://udlonline.cast.org/resources/ images/future_in_margins.pdf (consulta: 24/11/2014).

Rose, D. H. y Meyer, A. (2002). Teaching Every Student in the Digital Age: Universal Design for Learning. Alexandria, VA: Association for Supervision and Curriculum Development.

RuIz, D. (2013). La realidad aumentada y su aplicación en el patrimonio cultural. Trea, Colección Biblioteconomía y Administración Cultural.

Ruiz, D. (2011). Realidad aumentada, educación y museos. Revista icono 14(2), 212-226. ISSN 1697-8293.

SARracino, F. (2014). ¿Mejora la realidad aumentada el aprendizaje de los alumnos? Una propuesta de experiencia de museo aumentado. Profesorado: revista de currículum y formación del profesorado.

Vicent, N., Rivero, P. y Feliu, M. (2015). Arqueología y tecnologías digitales en Educación Patrimonial. Educatio Siglo XXI, 83-102.

Villar, S. (2012-2013). Aprendizaje Basado en Proyectos. Universidad de Zaragoza.

ORDEN del 20 de diciembre de 94 sobre la educación en valores (CV).

ORden 44/2011, de 7 de junio, de la Conselleria de Educación, por la que se regulan los planes para el fomento de la lectura en los centros docentes de la Comunitat Valenciana.

ReAl Decreto 126/2014, de 28 de febrero, por el que se establece el currículo básico de la Educación Primaria. 
ORDEN ECD/65/2015, de 21 de enero, por la que se describen las relaciones entre las competencias, los contenidos y los criterios de evaluación de la educación primaria, la educación secundaria obligatoria y el bachillerato. Evaluación.

ORDEN 32/2011, de 20 de diciembre, de la Conselleria de Educación, Formación y Empleo, por la que se regula el derecho del alumnado a la objetividad en la evaluación, y se establece el procedimiento de reclamación de calificaciones obtenidas y de las decisiones de promoción, de certificación o de obtención del título académico que corresponda.

ORDEN 89/2014, de 9 de diciembre, de la Conselleria de Educación, Cultura y Deporte, por la que se establecen los documentos oficiales de evaluación y se concretan aspectos de la ordenación general de la Educación Primaria en la Comunitat Valenciana. 